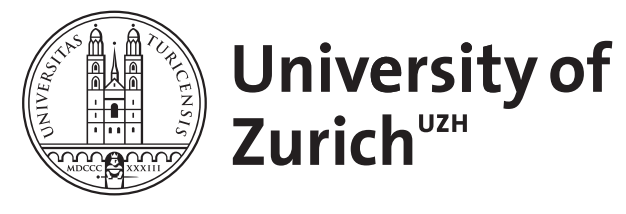

\title{
Altern und Sexualität
}

Bucher, T

\begin{abstract}
Dieser Beitrag gibt einen einführenden Überblick zum sexuellen Verhalten und Erleben von Menschen im mittleren und höheren Erwachsenenalter. Grundlage dafür ist eine Studie aus der deutschsprachigen Schweiz mit 641Männern (45-91Jahre) und 857Frauen (45-86Jahre). Altersbedingte hormonale und anatomische Veränderungen und deren Auswirkungen auf die sexuellen Reaktionen werden kurz beschrieben. Sexuelles Interesse, sexuelle Aktivität und Zufriedenheit, gesundheitliche Einflüsse darauf sowie Gründe sexueller Inaktivität werden dargestellt. Die Medikalisierung der Sexualität alternder Menschen wird kritisch beleuchtet. Zusammenfassende Empfehlungen für die Praxis werden gegeben
\end{abstract}

DOI: https://doi.org/10.1007/s00103-006-1271-2

Posted at the Zurich Open Repository and Archive, University of Zurich ZORA URL: https://doi.org/10.5167/uzh-156598

Journal Article

Published Version

Originally published at:

Bucher, T (2006). Altern und Sexualität. Bundesgesundheitsblatt, Gesundheitsforschung, Gesundheitsschutz, 49(6):567-574.

DOI: https://doi.org/10.1007/s00103-006-1271-2 
Bundesgesundheitsbl - Gesundheitsforsch Gesundheitsschutz 2006 - 49:567-574 DOI 10.1007/s00103-006-1271-2 Online publiziert: 28. April 2006 ๑) Springer Medizin Verlag 2006

\section{T. Bucher}

Psychologisches Institut, Sozial- und Gesundheitspsychologie, Universität Zürich, Zürich, Schweiz

\section{Altern und Sexualität}

A eines populären Buches über den Umgang mit Sexualität im Alter [1]. Ganz so einfach, wie es dieser Titel suggeriert, ist es jedoch nicht. Die körperlichen Grundlagen der Sexualität unterliegen wie alle anderen Organe und Körperfunktionen degenerativen Alterungsprozessen. Sexualität ist jedoch ein Prozess, der aus der Interaktion biologischer, psychologischer, sozialer, ökonomischer, politischer, kultureller, ethischer, gesetzlicher, historischer, religiöser und spiritueller Faktoren resultiert [2]. Deshalb lassen sich auf der Basis altersbedingter hormoneller, anatomischer und neuronaler Veränderungen keine zuverlässigen Prognosen über sexuelles Erleben und Verhalten ableiten. Andere Autoren sind der Meinung, dass der Begriff „Alterssexualität“ am besten abgeschafft würde ([3], S. 16) da er nahe legt, dass die Sexualität Älterer klar von der anderer Erwachsener abgrenzbar, essenziell vorgegeben und historisch unveränderbar sei, weil er Vielfalt einschränkt und suggeriert, dass die Sexualität im höheren Lebensalter vor allem durch das „physiologische" Merkmal Alter bestimmt sei.

Zwei Hauptschwierigkeiten sind damit angesprochen, die sich beim Thema Sexualität und Altern stellen: Wann beginnt das Alter oder das Altern? Und welches sind die Normen für die Sexualität alternder Menschen? Eindeutige Antworten gibt es auf beide Fragen nicht. Empirische Studien zur Sexualität alternder Menschen setzen meist bei 40 oder 45 Jahren an und umschreiben die untersuchte Zeitspanne, die meist bei 80 Jahren aufhört, mit mittlerem und höherem Erwachsenenalter, zweiter Lebenshälfte oder ähnlich. Diese Unschärfe kann auch dieser Beitrag nicht ausräu- men. „Ist es normal, mit 75 Jahren so oft sexuelle Lust zu haben?“ - „Wie häufig haben 70-Jährige noch Geschlechtsverkehr?“ Die Beantwortung solcher Fragen anhand von Mittelwertsangaben aus empirischen Studien bietet nur eine vermeintliche Orientierung. Die Varianz sexuellen Erlebens und Verhaltens wird mit zunehmendem Alter größer, die Aussagekraft von Durchschnittswerten entsprechend geringer [4, 5]. Statistisch gesicherte Normen zur Sexualität im Alter gibt es deshalb nicht.

\section{Statistisch gesicherte Normen zur Sexualität im Alter gibt es nicht}

Dieser Beitrag gibt einen einführenden Überblick zur Sexualität alternder Menschen. Zuerst werden altersbedingte körperliche Veränderungen beschrieben, die die Sexualität betreffen. Der Fokus richtet sich dann auf sexuelles Erleben und Verhalten. Diesbezügliche Grundlage sind die wichtigsten Forschungsergebnisse aus unserer Studie zu sexuellem Interesse, sexueller Aktivität und Zufriedenheit in der zweiten Lebenshälfte $[4,6]$. Es handelt sich dabei um eine Fragebogenstudie, an der 641 Männer und 857 Frauen aus der deutschsprachigen Schweiz im Alter zwischen 45 und 91 Jahren teilnahmen. Die Sexualität wurde im Rahmen von psychosozialen Ressourcen und Belastungen, Gesundheit und Partnerschaft untersucht. Die wichtigsten demographischen Merkmale der Stichprobe sind in - Tabelle 1 aufgeführt. Auf weitere methodische Aspekte wird hier nicht eingegangen, da diese bereits detailliert veröffentlicht sind $[4,6]$. Gesundheitliche Einflüsse auf sexu- elles Interesse und sexuelle Aktivität sowie Gründe für das Einstellen der koitalen Aktivität werden dargestellt. Im letzten $\mathrm{Ab}$ schnitt wird die „Medikalisierung“ der Sexualität im Alter kritisch thematisiert. Ein kurzes Resümee mit Empfehlungen für die Praxis schließt diesen Beitrag ab.

\section{Altersbedingte körperliche Veränderungen bei der Frau}

Die Wechseljahre beginnen bei Frauen in westlichen Gesellschaften etwa im 50. Altersjahr ( \pm 4 Jahre). Die Östrogenproduktion verringert sich um 90\%, der Menstruationszyklus wird unregelmäßig und bleibt schließlich ganz aus, womit die reproduktive Phase beendet ist. Begleitet werden die Wechseljahre oft von Beschwerden, die als klimakterisches Syndrom beschrieben werden: Hitzewallungen, Herzbeschwerden, Schlafstörungen, Stimmungsschwankungen (Depressivität, Nervosität), allgemeine Leistungsminderungen ( $\mathrm{Ge}$ dächtnis, Konzentration), Harnwegsbeschwerden, Trockenheit der Scheide sowie Gelenk- und Muskelbeschwerden [7]. Nicht alle Frauen sind davon gleichermaßen betroffen, rund 30-40\% haben kaum klimakterische Beschwerden [8]. Durch den dauerhaft tiefen Östrogenspiegel schrumpfen Eierstöcke, Eileiter, Uterus und Scheide. Die Vaginalhäute werden dünner, und die Scheidenschleimhaut wird trockener [9]. Als Folge davon können Schmerzen beim Geschlechtsverkehr auftreten. Der Lubrikationsmangel beim Geschlechtsverkehr kann aber auch auf mangelnde Stimulation oder psychische Ursachen wie fehlende Nähe zurückzuführen sein [10]. 
Tabelle 1

Soziodemographische Merkmale der Stichprobe

\begin{tabular}{|c|c|c|c|}
\hline & & $\begin{array}{l}\text { Frauen } \\
n=857\end{array}$ & $\begin{array}{l}\text { Männer } \\
\text { n=641 }\end{array}$ \\
\hline \multirow[t]{2}{*}{ Alter } & - Spanne & $45-86$ & 45-91 \\
\hline & •M(SD) & $58,2(8,9)$ & $61,8(10,5)$ \\
\hline \multirow[t]{3}{*}{ Altersgruppen ${ }^{a}$} & • 45-64 Jahre & $73,5 \%$ & $57,4 \%$ \\
\hline & • 65-84 Jahre & $26,4 \%$ & $40,6 \%$ \\
\hline & - 85 und mehr Jahre & $0,1 \%$ & $2,0 \%$ \\
\hline \multirow[t]{2}{*}{ Wohnort } & • Dorf/Kleinstadt & $64,7 \%$ & $68,4 \%$ \\
\hline & - Großstadt/Vorort & $35,3 \%$ & $31,6 \%$ \\
\hline \multirow[t]{4}{*}{ Zivilstand } & - Ledig & $9,4 \%$ & $6,4 \%$ \\
\hline & -Verheiratet & $56,8 \%$ & $72,1 \%$ \\
\hline & - Geschieden & $18,4 \%$ & $13,6 \%$ \\
\hline & •Verwitwet & $15,4 \%$ & $7,8 \%$ \\
\hline \multirow[t]{2}{*}{ Partnerschaft } & - Mit fester Partnerschaft & $70,5 \%$ & $86,0 \%$ \\
\hline & - Ohne feste Partnerschaft & $29,5 \%$ & $14,0 \%$ \\
\hline \multirow[t]{5}{*}{ Berufsbildung } & - Keine abgeschlossene Berufsbildung & $19,2 \%$ & $5,8 \%$ \\
\hline & - Berufslehre & $44,6 \%$ & $50,3 \%$ \\
\hline & - Fachschule, Lehrerseminar & $21,4 \%$ & $23,7 \%$ \\
\hline & • Hochschule & $4,9 \%$ & $12,9 \%$ \\
\hline & -Anderes & $9,9 \%$ & $7,3 \%$ \\
\hline \multirow[t]{4}{*}{ Berufliche Situation } & • Berufstätig & $40,4 \%$ & $47,7 \%$ \\
\hline & - Pensioniert & $30,3 \%$ & $46,3 \%$ \\
\hline & - Hausfrau/-mann & $23,9 \%$ & $0,5 \%$ \\
\hline & - Arbeitslos/anderes & $5,5 \%$ & $5,5 \%$ \\
\hline
\end{tabular}

a Da nur sehr wenige Untersuchungsteilnehmer älter als 85 Jahre sind, wird für die Darstellung von Ergebnissen von 45- bis 64-Jährigen (mittleres Erwachsenenalter) und 65-Jährigen und älteren (höheres Erwachsenenalter) unterschieden.

\section{Klimakterium und Sexualität}

Wie sich das Klimakterium auf die Sexualität auswirkt, ist unklar. In unserer Untersuchung berichten $41 \%$ der Frauen, dass ihr sexuelles Interesse während der Wechseljahre schwächer geworden ist, $51 \%$, dass es gleich geblieben ist, und $8 \%$, dass es zugenommen hat. Veränderungen in der Sexualität können jedoch nicht allein auf das Ausbleiben von Hormonen zurückgeführt werden. Negative Stereotypen über klimakterische oder postmenopausale Frauen sind weit verbreitet (Verlust von Weiblichkeit, negative Emotionen, Launenhaftigkeit) und können zu sich selbst erfüllenden Prophezeiungen führen $[11,12]$. Studien, die psychosoziale Variablen berücksichtigen, zeigen, dass Stress, psychische Gesundheit, Einstellungen, Beziehungs- und situationale Faktoren, teilweise Merkmale des Partners die Sexualität oft stärker beeinflussen als die Menopause selbst [13, 14, 15]. Einige
Frauen betonen gar die positiven Aspekte der Menopause: die Befreiung von Verhütungsproblemen, von der monatlichen Regelblutung sowie von der Angst vor unerwünschten Schwangerschaften [16].

\section{Körperliche Veränderungen beim Mann}

Die altersbedingten körperlichen Veränderungen verlaufen beim Mann graduell, einen klar markierten Einschnitt wie bei den Frauen gibt es nicht. Die hormonellen Veränderungen sind mit diagnostischem Aufwand mess-, für den Mann im Normalfall aber nicht spürbar. Etwa ab dem 4o. Altersjahr nimmt das im Blut frei verfügbare Testosteron jährlich um $1 \%$ ab, das Sexualhormone bindende Globulin nimmt $\mathrm{zu}$, wobei die individuellen Unterschiede sehr groß sind [17, 18]. Die wenigsten Männer entwickeln aufgrund der verringerten Hormonproduktion Symptome, die klinisch relevant werden
[19]. Nur ein Fünftel der Männer mit Hypogonadismus (Testosteronspiegel geringer als $12 \mathrm{nmol} / \mathrm{l}$ ) weisen eine PADAMSymptomatik ${ }^{1}$ auf [20].

$\mathrm{Ab}$ dem 65. Lebensjahr treten erste degenerative Veränderungen in Samenkanälchen des Hodens auf, die Elastizität der Samenwege lässt nach, in Nebenhoden und anderen Geschlechtsdrüsen werden Dysfunktionen häufiger. Die Spermienproduktion nimmt ab, die Qualität der Spermien wird schlechter (geringere Motilität, mehr Missbildungen), und das Volumen des Ejakulats wird kleiner. Die häufig auftretende gutartige Vergrößerung der Prostata kann unter Umständen Probleme bei der Harnentleerung und beim Ejakulieren mit sich bringen [9]. In der Regel bleibt der Mann aber bis ins höchste Alter zeugungsfähig.

\section{Veränderung der sexuellen Reaktionen beim Mann}

Etwa ab dem 40. Altersjahr verändern sich die sexuellen Reaktionen beim Mann langsam, aber merklich: Es dauert länger, bis eine Erektion zustande kommt, sie ist weniger hart, und manchmal fehlt sie ganz. Mehr und stärkere Stimulation ist notwendig, um eine Erektion zu erreichen. Der Drang zu ejakulieren ist kleiner, die Kontrolle über die Ejakulation besser. Der Orgasmus ist weniger intensiv; die BulbourethralDrüsen produzieren bei sexueller Erregung weniger Sekret (Lusttropfen); der Penis erschlafft nach der Ejakulation schneller, und die Refraktärphase ist länger [17].

Diese Veränderungen werden unterschiedlich wahrgenommen: Für manche Männer sind sie eine Kränkung, da sie nicht ihrem Selbstbild als Mann entsprechen. Andere nehmen sie gelassen hin oder sehen darin sogar Vorteile, da die verlangsamten Reaktionen und die bessere ejakulatorische Kontrolle den Bedürfnissen der Partnerin entgegenkommen [21]. Wie die Reaktionen auf die altersbedingten Veränderungen aus-

\footnotetext{
${ }^{1}$ PADAM=Partielles Androgendefizit des alternden Mannes. Die Symptome sind Müdigkeit, Abgeschlagenheit, Stimmungsschwankungen, Depressionen, Kraftlosigkeit, Muskelschwund, Bildung von Fettpolstern in der Hüftgegend, Gewichtszunahme, Vergrößerung der Brustdrüsen, Nachlassen der Libido, selten auch Erektionsstörungen und Gliederschmerzen durch Osteoporose [19].
} 


\section{Zusammenfassung $\cdot$ Abstract}

fallen, hängt von verschiedenen Faktoren ab: Wichtigkeit der Sexualität für Selbstwert, Wohlbefinden und Partnerschaft, Angemessenheit von Standards oder Referenzgruppen für die eigenen sexuellen Reaktionen, Fähigkeit, sich auf erotische Stimuli zu konzentrieren und nicht durch Selbstbeobachtung die Angst vor Versagen zu verstärken, Häufigkeit und Bandbreite von sexueller Aktivität in jüngeren Jahren, Motivation und Fähigkeit, andere Praktiken als nur vaginalen Geschlechtsverkehr auszuprobieren, unterstützende Haltung und funktionierende sexuelle Kommunikation mit der Partnerin [17].

\section{Gewünschte und erlebte sexuelle Aktivität}

Als Indikator für die Sexualität wird in vielen Studien lediglich die Koitusfrequenz verwendet. Diese Reduktion auf eine Sexualpraktik ist wenig zutreffend für die Sexualität älterer Menschen [5]. Deshalb haben wir in unserer Studie zwischen sexuellem Interesse, sexueller Aktivität und Zufriedenheit unterschieden $[4,6]$.

\section{Sexuelles Interesse}

Die sexuellen Wünsche untersuchten wir mit der Frage: „Stellen Sie sich vor, Sie könnten wünschen. Wie häufig hätten Sie gerne, dass Folgendes vorkommen würde?" Die Teilnehmer/-innen konnten sich zu Zärtlichkeiten im Alltag wie Streicheln, in den Arm nehmen oder Küssen, Petting, d. h. Schmusen, Streicheln und sexuelle Stimulation, ohne anschließend Geschlechtsverkehr zu haben, sowie Geschlechtsverkehr äußern.

\section{( ) Bei Männern hat der Partner- schaftsstatus anders als bei Frauen keinen Einfluss auf die sexuellen Wünsche}

Die Korrelationen in • Tabelle 2 zeigen, dass die gewünschte Häufigkeit von sexuellen Aktivitäten mit steigendem Alter abnimmt; am deutlichsten trifft dies für den Wunsch nach Geschlechtsverkehr zu. Sexuelle Aktivitäten werden weniger oft gewünscht, die Wünsche bleiben aber bei sehr vielen Menschen grundsätzlich bis ins höchste Alter erhalten (• Abb. 1). Ist

Bundesgesundheitsbl - Gesundheitsforsch - Gesundheitsschutz 2006 • 49:567-574

DOI 10.1007/s00103-006-1271-2

○) Springer Medizin Verlag 2006

\section{T. Bucher}

\section{Altern und Sexualität}

\section{Abstract}

Dieser Beitrag gibt einen einführenden Überblick zum sexuellen Verhalten und Erleben von Menschen im mittleren und höheren Erwachsenenalter. Grundlage dafür ist eine Studie aus der deutschsprachigen Schweiz mit 641 Männern (45-91 Jahre) und 857 Frauen (45-86 Jahre). Altersbedingte hormonale und anatomische Veränderungen und deren Auswirkungen auf die sexuellen Reaktionen werden kurz beschrieben. Sexuelles Interesse, sexuelle Ak-

\section{Aging and sexuality}

\section{Abstract}

This contribution describes sexual desire, activity and satisfaction of people aged 4591 years on the basis of an empirical study conducted in the German-speaking part of Switzerland. Sexual desire and activity diminish with age but there is a great variability. Sexual satisfaction remains stable in elderly people. Physical, hormonal and neuronal changes cause a greater vulnerability for sexual problems but relationship, psy- tivität und Zufriedenheit, gesundheitliche Einflüsse darauf sowie Gründe sexueller Inaktivität werden dargestellt. Die Medikalisierung der Sexualität alternder Menschen wird kritisch beleuchtet. Zusammenfassende Empfehlungen für die Praxis werden gegeben.

\section{Schlüsselwörter}

Sexualität · Alter · Sexuelle Zufriedenheit . Medikalisierung

chosocial and biographical factors as well as coping strategies have a strong moderating effect. The medicalization of the sexuality of aging people is critically depicted. Recommendations for health care practitioners are given.

\section{Keywords}

Sexuality · Aging · Sexual satisfaction .

Medicalization 


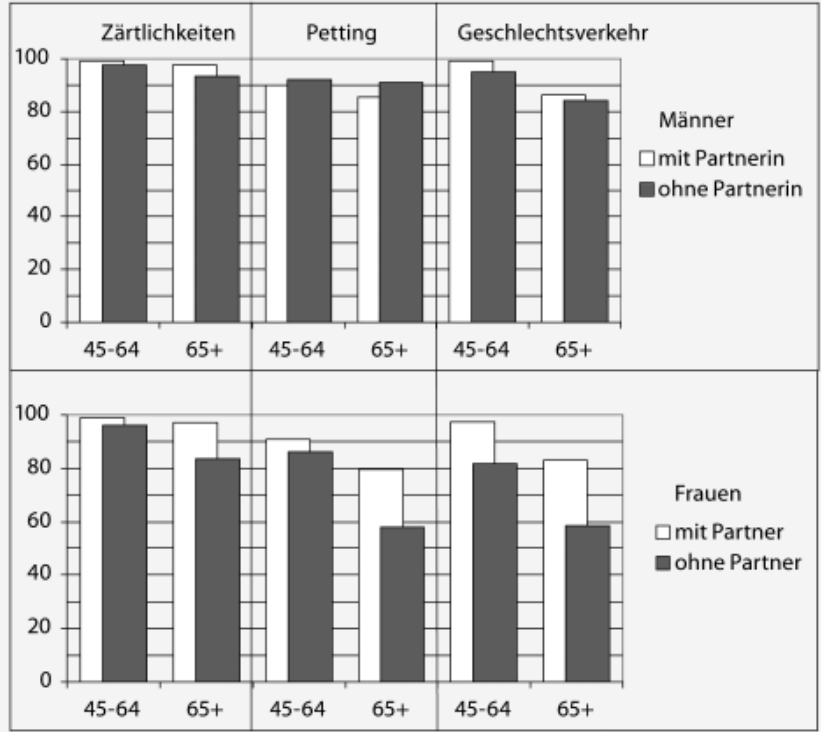

Abb. $1<$ Sexuelle Wünsche nach Altersgruppe und Partnerstatus (Prozentangaben für Personen mit entsprechendem Wunsch)

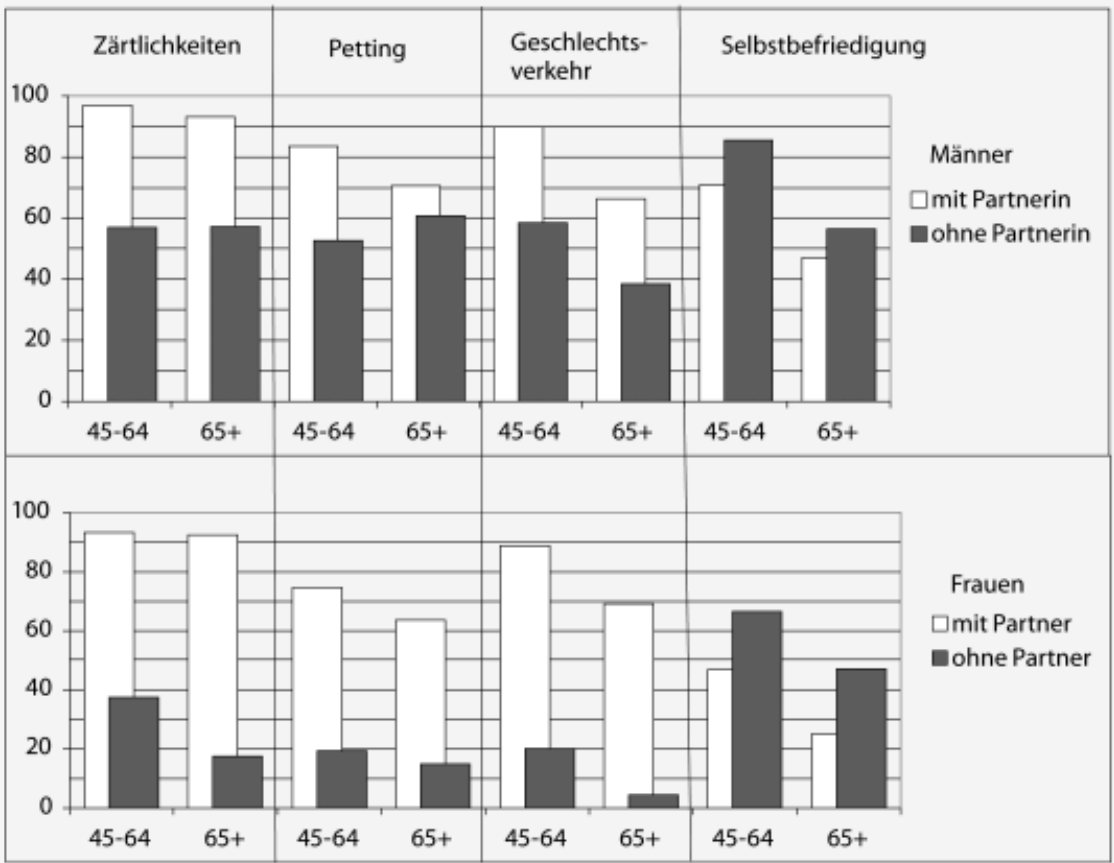

Abb. $2 \triangle$ Sexuelle Aktivitäten nach Altersgruppe und Partnerschaftsstatus (Prozentangaben für Personen mit entsprechendem Verhalten)

eine feste Partnerschaft vorhanden, trifft dies für Männer wie Frauen gleichermaßen zu. Frauen der älteren Gruppe ohne festen Partner haben öfter kein sexuelles Interesse, bei den Männern hat der Partnerschaftsstatus keinen Einfluss auf die sexuellen Wünsche. Wie kann dieser Unterschied erklärt werden? Der Partnermangel aufgrund demographischer Verhältnisse ist sicher ein Grund: Ohne Aussicht auf einen akzeptablen Partner bringt es mehr Frustration als Befriedigung mit sich, große sexuelle Bedürfnisse zu hegen.
Diese Erklärung genügt jedoch nur teilweise. Die Frageformulierung (stellen Sie sich vor, Sie könnten wünschen ...) würde erlauben, diese Anpassung an die Umstände zu ignorieren. Vermutlich stehen hier mehr kohorten- als altersspezifische Gründe im Vordergrund. Die meisten Frauen der älteren Generation wurden dahingehend sozialisiert, sich Kinder, nicht aber eine lustvolle und befriedigende Sexualität zu wünschen. Viele von ihnen gingen Versorgungsehen ein, in denen Liebe und Zärtlichkeit eine geringe Rolle spielten und die
Sexualität eheliche Pflicht zwecks Familiengründung und Befriedigung des Mannes war. Alter und Menopause sind für viele Frauen dieser Generation deshalb ein willkommener Vorwand, die Sexualität einstellen zu können $[5,16]$. Die Frauen im mittleren Lebensalter hingegen erlebten die sexuelle Revolution in jungen Jahren („Pille“ und Liberalisierung) und konnten von den Errungenschaften der Frauenbewegung profitieren. Das ermöglichte ihnen, gleichberechtigte und selbstbestimmte (sexuelle) Beziehungen einzugehen (oder sie aufzulösen, falls sie nicht ihren Vorstellungen entsprachen). Diese Frauen können ihre sexuellen Bedürfnisse heute auch ausdrücken. Es ist anzunehmen, dass dieser Anteil an selbstbestimmten Frauen zunehmen wird [16].

\section{Sexuelle Aktivität}

Neben den partnerbezogenen sexuellen Aktivitäten haben wir in unserer Studie auch die Selbstbefriedigung berücksichtigt. Die Korrelationen für die partnerbezogenen sexuellen Aktivitäten sind mit denen des sexuellen Interesses vergleichbar: Mit zunehmendem Alter werden sie weniger häufig praktiziert, am stärksten trifft dies wiederum auf den Geschlechtsverkehr zu (• Tabelle 2). Der Einfluss des Alters auf die sexuelle Aktivität darf aufgrund von Korrelationen jedoch nicht überschätzt werden. Das Alter ist stark mit der Partnerschaftsdauer konfundiert. Bis zum 6o. Altersjahr verringert die Partnerschaftsdauer die sexuelle Aktivität stärker als das Alter [22].

Wird der Partnerschaftsstatus berücksichtigt, ergeben sich Differenzierungen: Ist eine feste Partnerschaft vorhanden, sind keine bedeutenden Unterschiede zwischen Männern und Frauen zu beobachten (• Abb. 2). Zärtlichkeiten kommen in beiden Altersgruppen sehr häufig vor, während Petting und Geschlechtsverkehr in der älteren Gruppe häufiger fehlen. Ist keine Partnerschaft vorhanden, sind partnerbezogene sexuelle Aktivitäten deutlich seltener, bei den Frauen sind sie dann die Ausnahme. Mehr Alleinstehende als fest Liierte praktizieren Selbstbefriedigung, für Frauen ohne festen Partner ist sie die häufigste Sexualpraktik.

Die Ergebnisse zeigen, dass eine feste Partnerschaft die wesentliche Vorausset- 
zung ist, um im mittleren und höheren Alter das Bedürfnis nach Zärtlichkeiten, Petting und Geschlechtsverkehr leben zu können. Aufgrund der kürzeren Lebenserwartung der Männer und der Altersdifferenz zwischen Ehepartnern (die Frauen sind meist jünger) ist für Frauen diese Voraussetzung mit steigendem Alter immer weniger gegeben. Aus denselben demographischen Gründen ist es für allein stehende Frauen sehr schwierig, einen passenden Partner zu finden, während dies den Männern eher zu gelingen scheint: Immerhin etwa die Hälfte berichtete über sexuelle Aktivitäten mit einer Partnerin.

\section{Gesundheitliche Einflüsse auf sexuelles Interesse und sexuelle Aktivität}

Verschiedene Gesundheitsindikatoren (subjektive Gesundheit, Beschwerden, Medikamentenkonsum) weisen bei Männern stärkere Korrelationen mit sexuellem Interesse und sexueller Aktivität auf als bei Frauen [4, $6,23]$. Vermutlich spielen hier Krankheiten eine wesentliche Rolle, die durch einen ungesunden Lebensstil verstärkt werden und sich negativ auf die Erektionsfähigkeit auswirken (Alkohol- und Nikotinkonsum, Bewegungsmangel und Übergewicht). Folgende Krankheiten und Medikamente werden mit Libidoverlust und Erektionsstörungen in Verbindung gebracht: Herz-Kreislauf-Erkrankungen, Bluthochdruck, Diabetes, Nieren- und Leberinsuffizienz, Störungen der Nebennierenfunktionen (Addison-Krankheit, Cushing-Syndrom), Adipositas, Störungen der Schilddrüsen, Arteriosklerose, Arthritis, Parkinson, multiple Sklerose, Alzheimer, Depression, Hirnschlag. Medikamente: Bluthochdruckmittel, Herz-Kreislauf-Medikamente, Beruhigungsmittel, Psychopharmaka, Entwässerungsmittel (Diuretika), Kortison, Mittel zur Magenentsäuerung $\left(\mathrm{H}_{2}\right.$-Antagonisten) und Antiandrogene $[17,24]$.

Altersbedingte Gebrechen, chronische Krankheiten, allgemeine Schwäche und die Folgen von Operationen vermindern das sexuelle Verlangen und die sexuelle Aktivität auch bei Frauen. Die Gesundheit scheint aber für Frauen eine weniger große Rolle zu spielen als für Männer. Die Korrelationen mit unterschiedlichen Gesundheitsindikatoren sind ziemlich gering $[4,25,26]$.

Tabelle 2

Sexuelles Interesse, sexuelle Aktivität und Zufriedenheit: Korrelationen mit dem Altera

\begin{tabular}{|lll}
\hline & Männer & Frauen \\
\hline Wunsch nach Zärtlichkeiten & -.24 & -.29 \\
\hline Wunsch nach Petting & -.22 & -.30 \\
\hline Wunsch nach Geschlechtsverkehr & -.53 & -.40 \\
\hline Häufigkeit von Zärtlichkeiten & -.20 & -.29 \\
\hline Häufigkeit von Petting & -.18 & -.21 \\
\hline Häufigkeit von Geschlechtsverkehr & -.45 & -.40 \\
\hline Selbstbefriedigung & -.33 & -.16 \\
\hline Sexuelle Zufriedenheit & -.18 & -.07 (n.s.)
\end{tabular}

${ }^{\text {a } W e n n ~ n i c h t ~ a n d e r s ~ v e r m e r k t: ~} p<.01$.

Tabelle 3

Gründe für sexuelle Inaktivität (Mehrfachantworten waren möglich)

\begin{tabular}{lcc} 
Grund & Männer & Frauen \\
\hline Kein(e) Partner(in) vorhanden & $21,9 \%$ & $60,4 \%$ \\
\hline Partner(in) kann nicht mehr (Alter, Krankheit etc.) & $18,2 \%$ & $18,7 \%$ \\
\hline Ich habe keine Lust mehr & $14,6 \%$ & $19,4 \%$ \\
\hline Anderes & $17,5 \%$ & $13,9 \%$ \\
\hline Ich bin zu alt dazu & $9,5 \%$ & $6,2 \%$ \\
\hline Partner(in) will nicht mehr & $29,9 \%$ & $6,2 \%$ \\
\hline Ich kann nicht mehr (Impotenz) & $20,4 \%$ & a \\
\hline Es bereitet mir Schmerzen & $0,7 \%$ & $4,0 \%$ \\
\hline Partner(in) ist zu wenig attraktiv & $2,2 \%$ & $1,5 \%$ \\
\hline Ich bin zu wenig attraktiv & $0,0 \%$ & $1,8 \%$
\end{tabular}

${ }^{a}$ Diese Antwort konnte nur von Männern gegeben werden.

\section{Gründe für die sexuelle Inaktivität}

Bisherige Untersuchungen zeigen, dass das Alter per se selten der Grund für sexuelle Inaktivität ist. Der Partnermangel ist meist dafür verantwortlich. Bei Paaren sind die Ursachen häufiger beim Mann als bei der Frau zu suchen: Gesundheitliche Gründe sowie Erektionsprobleme werden am häufigsten genannt $[5,13,16,27]$. Ähnliche Ergebnisse finden wir in unserer Studie. 137 Männer (45-89 Jahre; $M=68,6$ Jahre) und 237 Frauen (45-86 Jahre; $M=62,5$ Jahre) beantworteten die Frage, weshalb sie in den 3 Monaten vor der Befragung keinen Geschlechtsverkehr hatten (• Tabelle 3). Für Frauen ist der Hauptgrund der Partnermangel (über 60\%), gefolgt von der eigenen Lustlosigkeit und Gründen, die beim Partner liegen (Alter, Krankheit etc.). Männer gaben am häufigsten an, dass die Partnerin nicht mehr will (30\%), Partnermangel und Impotenz (je ca. 20\%) sind weitere oft genannte Gründe. Weniger als 10\% machen ihr Alter für die koitale Inaktivität verantwortlich. Zur Antwortkategorie „Anderes“ konnten zusätzliche Angaben gemacht werden. Die Antworten unterscheiden sich stark je nach Geschlecht. Die meisten Männer führen hier gesundheitliche Probleme an, sehr selten erwähnen sie aktuelle Beziehungsschwierigkeiten. Frauen geben differenziertere Antworten, die meist auf ihre soziale Situation als Frau verweisen. Die inhaltsanalytische Auswertung ergab 6 Kategorien. Sie sind stichwortartig mit je einem Beispiel in - Tabelle 4 aufgeführt. Nicht alle Kategorien sind geschlechts-, alters- oder generationenspezifisch. Die traditionelle Sexualmoral beispielsweise richtet sich an alle; nur ist 
Tabelle 4

Gründe für sexuelle Inaktivität: 6 Kategorien in offenen Antworten

von Frauen

1. Missbrauchserfahrungen

53-jährig, ohne Partner, seit 7 Jahren geschieden und sexuell inaktiv:

"Nach sehr langer Beziehung mit einem Gewalttätigen habe ich weder das Vertrauen zu einem

Mann gefunden, noch irgendetwas empfunden. Ein Ekel, Angst vor Gewalt, Ausnutzung oder

Nichtbestehenbleiben der Beziehung."

2. Traditionelle Rollenaufteilung in der Partnerschaft

57-jährig, seit 36 Jahren verheiratet, seit 5 Monaten sexuell inaktiv:

„Ich bin für meinen Mann nur noch Haushälterin, Sekretärin, Kindermädchen. Mit denen geht

man nicht ins Bett. Das ist meine Begründung, dass ich keine Lust mehr habe. Ein anderer Mann kommt nicht in Frage wegen des Dorfklatsches. Daran habe ich auch schon gedacht."

3. Befolgung traditioneller Normen

75-jährig, seit 30 Jahren geschieden, seither ohne sexuelle Kontakte:

„Als gläubige Christin gibt es für mich keinen Sexualverkehr, solange ich nicht verheiratet bin."

4. Bewahrung der Unabhängigkeit durch Verzicht auf Sexualität

72-jährig, ledig, lebt mit ihrer Schwester zusammen, ohne sexuelle Beziehungen:

"Ich wollte berufstätig bleiben und kein ,Spielzeug' werden."

5. Sexualität ist nicht Bestandteil des Selbstbildes alter Frauen

75-jährig, seit 26 Jahren verwitwet, ohne Zeitangaben zur sexuellen Inaktivität:

"ICh bin eine 75-jährige Frau!"

6. Steigende Ansprüche an situativen und partnerschaftlichen Kontext

63-jährig, seit 22 Jahren geschieden, ohne Partner, seit 11 Jahren sexuell inaktiv:

„Man könnte schon Sex haben, aber je älter man wird, genügt Sex alleine nicht; es muss alles

stimmen. Man wird sehr wählerisch und anspruchsvoll."

sie für Frauen viel restriktiver und wurde wie die anderen Kategorien in diesem $\mathrm{Zu}$ sammenhang nur von Frauen genannt. Lediglich eine Antwort eines verwitweten, 54jährigen Mannes, der seit 9 Jahren keine sexuellen Kontakte mehr hatte, verweist auf das soziale Geschlechterverhältnis. Sie deutet jedoch darauf hin, dass er mit der sich wandelnden Rolle der Frau Schwierigkeiten hat. Er schrieb: „Es gibt keine unkomplizierten, normal denkenden Frauen mehr. Alle, nicht nur Frauen, versuchen nur noch, ihre Zwänge auszuleben.“

\section{Das Alter per se ist selten der Grund für sexuelle Inaktivität}

Insgesamt zeigt sich, dass der Partnermangel der Hauptgrund für das Einstellen der sexuellen Aktivität ist. Nur in wenigen Fällen ist das Alter für das sexuelle Disengagement der Grund. Körperliche Ursachen werden eher von Männern genannt, während Frauen öfter biographische Erfahrungen aufgrund ihrer gesellschaftlich definierten Rolle und Stellung anführen. $\mathrm{Ob}$ sich dies in Zukunft ändern wird, wenn die bei Jugendlichen beobachtete Anglei- chung im Sexualverhalten auch die älteren Generationen erreicht, kann zum heutigen Zeitpunkt nicht gesagt werden.

\section{Sexuelle Zufriedenheit}

Die sexuelle Zufriedenheit wurde in unserer Untersuchung mit einer 5-stufigen Skala gemessen ( $1=$ sehr unzufrieden bis $5=$ sehr zufrieden). Übereinstimmend mit anderen Studien $[17,28]$ finden wir nur schwache oder nicht signifikante Korrelationen mit dem Alter (• Tabelle 2). Die sexuelle Zufriedenheit verringert sich also nicht im gleichen Maß wie das sexuelle Interesse oder die sexuelle Aktivität. Dieser Befund wird einerseits mit der parallelen Abnahme von sexuellem Interesse und sexueller Aktivität erklärt [17]. Andererseits gewinnen im mittleren und höheren Erwachsenenalter Beziehungsaspekte für die sexuelle Zufriedenheit an Bedeutung, nur werden solche in der Forschung selten berücksichtigt. Lediglich der Zusammenhang zwischen Partnerschaftszufriedenheit und sexueller Zufriedenheit wurde mehrmals bestätigt, ohne eine kausale Richtung bestimmen zu können [29].
Mittels Strukturgleichungsmodellen konnten anhand unserer Daten die Gesamteffekte von partnerschaftlichen Merkmalen auf die sexuelle Zufriedenheit untersucht werden [30]. Dafür wurden die Angaben von 400 Männern (45-87 Jahre, $M=58,5$ Jahre) und 443 Frauen (45-78 Jahre; $\mathrm{M}=54,8$ Jahre) zu ihrer partnerschaftlichen Situation verwendet. Die Resultate brachten einige überraschende Geschlechtsunterschiede. Die bedeutsamen Prädiktoren für die sexuelle Zufriedenheit waren für die Männer das Kommunizieren der eigenen sexuellen Wünsche gegenüber der Partnerin $(.35)^{2}$, die Zurückhaltung der Partnerin in sexuellen Belangen (-.28), die sexuelle Aktivität (d. h. Zärtlichkeiten, Petting und Geschlechtsverkehr; .27), das sexuelle Interesse (.17) sowie die Überforderung durch die sexuellen Ansprüche der Partnerin (-.12). Dieses Ergebnis betont die Wichtigkeit des Austauschs über die eigenen sexuellen Bedürfnisse und weist auch auf widersprüchliche Entwicklungen hin: Die zurückhaltende Partnerin (als negativer Einfluss) hört sich nach einer typischen Männerklage an. Grund dafür ist vermutlich die Retraditionalisierung von Geschlechterrollen im Verlaufe lang dauernder Beziehungen: Der Mann wünscht sich häufiger Geschlechtsverkehr, während die Frau mehr Zärtlichkeiten (ohne koitale Aktivität) möchte [22]. Männer nehmen in der Folge ihre Partnerin als zurückhaltend wahr, die Diskrepanz in den Wünschen führt zu Unzufriedenheit. Die Überforderung durch die sexuellen Ansprüche der Partnerin scheint dazu ein Widerspruch zu sein. Vermutlich führen zu große (Leistungs-)Erwartungen bei Männern zu Versagerängsten, was besonders zutrifft, wenn die Partnerin wesentlich jünger ist. Dies ist in Beziehungen nach einer ersten Ehe oft der Fall und verschärft sich, wenn ungelöste Probleme oder ausstehende Entwicklungsschritte von einer Beziehung in die nächste mitgenommen werden $[23,30]$.

Für die Frauen erwiesen sich folgende Prädiktoren als bedeutsam: die emotionale Nähe in der Partnerschaft (.28), das Ausdrücken der eigenen sexuellen Wünsche

\footnotetext{
${ }^{2}$ Die Zahlen in den Klammern sind die totalen Effekte auf die sexuelle Zufriedenheit, die mittels Strukturgleichungen berechnet wurden. Sie zeigen die relative Stärke des Effekts und dessen positive oder negative Richtung an.
} 
gegenüber dem Partner (.26), die Zurückhaltung des Partners in sexuellen Belangen (-.26), die sexuelle Aktivität (.24), die physische Attraktivität des Partners und das eigene sexuelle Interesse (je .12). Die Resultate zeigen, dass eine gute Beziehung eine wichtige Voraussetzung für eine zufrieden stellende Sexualität von Frauen im mittleren und höheren Lebensalter ist. Das Ausdrücken der eigenen sexuellen Wünsche weist auf die sexuelle Selbstbestimmung hin, die insbesondere für die älteren Frauen keine Selbstverständlichkeit ist (vgl. auch Abschnitt zum sexuellen Interesse). Die Zurückhaltung des Partners in der Sexualität ist vermutlich ein Hinweis auf zunehmende Erektionsprobleme des Mannes. Dabei ist die schwache oder fehlende Erektion für die Frau oft nicht das primäre Problem, sondern die Reaktion des Mannes darauf: Aus Scham oder Angst wird die körperliche Intimität mit der Partnerin oft gänzlich eingestellt, sodass Zärtlichkeiten und körperliche Nähe völlig fehlen und zu Frustrationen führen.

\section{(7) Die Sexualität der Frau wird viel stärker von sozialen, situationa- len und partnerschaftlichen als von physiologischen und hormo- nellen Merkmalen bestimmt}

Überraschend ist die Bedeutung der physischen Attraktivität des Partners (ein stärkeres Reagieren auf visuelle Reize ist sonst bei Männern zu beobachten). Sie hat bei den Frauen einen direkten Einfluss auf das sexuelle Interesse und die sexuelle Zufriedenheit, während sie bei den Männern nur das sexuelle Interesse direkt verstärkt. Das Ergebnis macht deutlich, dass sexuelle Stimuli in einem adäquaten sozialen und partnerschaftlichen Kontext für die sexuelle Motivation und Erregung von Frauen eine herausragende Rolle spielen. Das stützt Befunde, wonach die Sexualität der Frau viel stärker von sozialen, situationalen und partnerschaftlichen Merkmalen als von physiologischen und hormonellen Faktoren bestimmt wird [31], insbesondere bei Frauen in länger dauernden Beziehungen. Diese Aspekte wurden in der Forschung lange vernachlässigt und finden erst langsam die Beachtung, die ihnen zukommen sollte $[32,33]$.

Die Ergebnisse zeigen, dass kommunikative und partnerschaftliche Aspekte für die sexuelle Zufriedenheit im mittleren und höheren Lebensalter genauso bedeutend sind wie die sexuelle Aktivität.

\section{Medikalisierung der Sexualität und neue Normen}

Dieser letzte Abschnitt weist auf gesellschaftliche Tendenzen hin, die aus sozialwissenschaftlicher Sicht als „Medikalisierung“ der Sexualität bezeichnet werden. Sie stehen in Zusammenhang mit den Werten (und Möglichkeiten) der postmodernen Leistungsgesellschaft, von denen die Sexualität nicht verschont bleibt, auch die alternder Menschen nicht.

Die Verminderung der körperlichen Funktions- und Leistungsfähigkeit ist Teil des normalen Alterungsprozesses. Davon ist auch die Sexualität betroffen. Die daraus resultierende Anpassung von Erwartungen an die körperlichen Möglichkeiten, Neubewertungen und die Entwicklung von Alternativen ist eine Entwicklungsaufgabe dieses Lebensabschnitts. Die hohe durchschnittliche Lebenserwartung bei oft guter Lebensqualität in westlichen Gesellschaften hat zur Folge, dass (fast) alle alt werden wollen, nur niemand alt sein will: Vitalität, Jugendlichkeit und Fitness, Mobilität und Flexibilität sind auch für Senioren hoch gehaltene Werte, die mit einem breiten Anti-aging-Angebot bedient werden. Kosmetik und Hormone, Chemie und Chirurgie, Fit- und Wellnesskuren je nach Geschmack mit Ayurveda oder Antioxidanzien sollen das Altern verlangsamen oder gar verhindern. Degeneration, Abhängigkeit und Tod werden verdrängt, die Menschen sollen dem Markt als alterslose Konsumenten möglichst lange erhalten bleiben.

Galt früher das Vorurteil vom asexuellen Alter, so ist heute ein aktives Sexualleben zum Indikator für erfolgreiches Altern und somit zur neuen Norm für gesundheitsbewusste Senioren und Seniorinnen geworden. Altersbedingte Veränderungen in der Sexualität werden deshalb oft von medizinischer Seite zu „sexuellen Dysfunktionen “ umdefiniert, zu organisch bedingten Problemen, die losgelöst von Alter und psychosozialer Situation diagnostiziert und therapiert werden können [34]. Sehr deutlich lässt sich dieser Prozess anhand der erektilen Dysfunktion und der sexuellen Lustlosigkeit bei
Frauen veranschaulichen. Der Kölner Erfassungsbogen zur erektilen Dysfunktion setzt einen altersunabhängigen Cutt-OffWert fest, der zwischen Männern mit und ohne erektile Dysfunktion unterscheidet [35]. Ein 80-jähriger Mann muss somit die gleiche erektile Kapazität aufweisen wie ein 30-jähriger, damit er nicht als dysfunktional klassifiziert wird. Ähnlich werden Frauen, die nicht ein willkürlich festgelegtes Mindestmaß an sexueller Lust aufweisen, mit der Diagnose „female sexual dysfunction“ belegt. In einer österreichischen Studie mussten 20- bis 80-jährige Frauen im Zeitraum von 4 Wochen vor dem Befragungszeitpunkt mindestens „oft“ (frequently) oder „sehr oft “ Verlangen nach sexueller Aktivität aufweisen, um nicht der Gruppe mit „desire disorder“ zugeteilt zu werden [36]. Solches Vorgehen generiert Prävalenzraten von sexuellen Dysfunktionen, die bei 60 - bis 80 -Jährigen zwischen $50 \%$ und $100 \%$ liegen. Leonore Tiefer karikierte die Konsequenzen dieser auf das organische Funktionieren reduzierten Sichtweise sehr treffend: „The man's pill makes the women need a lubricant. The woman's lubricant makes the man need a penile sensory enhancer. The man's sensory enhancer makes the woman need a desire additive. The woman's desire additive makes the man need an energy stimulant. The man's energy stimulant makes him need antianxiety medication. And so on “ [37].

Die Fortschritte in der Behandlung körperlich bedingter sexueller Störungen sollen hier nicht einseitig schlecht gemacht werden. Sie bringen Hilfe, Erleichterung und eine Steigerung der Lebensqualität für viele Patienten. Die Verschreibung von $\mathrm{Me}$ dikamenten, die die Sexualität beeinflussen, sollte aber mit Blick auf den ganzen Menschen, einschließlich seines/ihres Alters, seiner/ihrer psychosozialen und vor allem auch partnerschaftlichen Situation geschehen und nicht nur hinsichtlich maximaler organischer Funktionstüchtigkeit.

\section{Resümee}

„Alte Liebe rostet nicht.“ Dieser Satz trifft $\mathrm{zu}$ - braucht aber Differenzierung. Altersbedingte hormonale, anatomische und neuronale Veränderungen vermindern und verlangsamen die sexuellen Reaktionen. Das bringt eine erhöhte Vulnerabili- 
tät für sexuelle Störungen und Unzufriedenheit mit sich, die jedoch nicht eintreffen müssen. Biographische, psychosoziale und partnerschaftliche Faktoren, Anpassung von Erwartungen sowie Copingstrategien moderieren in starkem Maß eine vermeintlich einseitig degenerative Entwicklung im sexuellen Erleben und Verhalten. Die Annahme über eine generelle Verminderung der sexuellen Zufriedenheit im höheren Lebensalter trifft deshalb nicht zu.

Über die sexuellen Probleme und Schwierigkeiten im Alter wissen wir relativ wenig. Die meisten diesbezüglichen Studien orientieren sich am Phasenmodell sexueller Reaktionen von Masters und Johnson (desire, arousal, orgasm) und den daraus abgeleiteten sexuellen Dysfunktionen. Sie geben entsprechende standardisierte Antworten vor (z. B. [26]) und berücksichtigen psychosoziale Zusammenhänge und die subjektive Bedeutungen der Sexualität im Lebenskontext der Individuen nur wenig. Die offenen Antworten auf die Frage nach den Gründen für das Einstellen der koitalen Aktivität (- Tabelle 4) zeigen jedoch deutlich, dass biographische und soziale Dimensionen unter besonderer Berücksichtigung der Geschlechterperspektive vermehrter Berücksichtigung bedürfen. Hier wären explorierende, qualitative Studien notwendig.

Partnerschaft und Sexualität haben für viele ältere Menschen mit Blick auf die allgemeine Lebenszufriedenheit einen großen Stellenwert. Damit sind oft hohe Erwartungen verbunden - gespeist aus den Erfahrungen in jüngeren Jahren, medial vermittelten Bildern und den Versprechungen der Pharmaindustrie. Häufig fehlt aber das Wissen über altersbedingte Veränderungen und wie damit umgegangen werden kann. In der Praxis ist es deshalb wichtig, auf normale Altersentwicklungen und „Störanfälligkeiten“ aufmerksam zu machen. Insbesondere sollte auf die dämpfende Wirkung von Medikamenten hingewiesen und falls möglich nach Alternativen gesucht werden. Die pharmakologische Therapie von Sexualstörungen ist kein Ersatz für ein Gespräch über sexuelle Probleme. Die genaue medizinische Abklärung von Kontraindikationen, Risiken und Nebenwirkungen, ein vertrauensvolles Gespräch und der Einbezug von Partner oder Partnerin gehören unbedingt zur Beratung und Behandlung von älteren Menschen mit sexuellen Problemen.

\section{Korrespondierender Autor T. Bucher}

Psychologisches Institut, Sozial- und Gesundheitspsychologie, Universität Zürich, Rämistrasse 66, 8001 Zürich, Schweiz

E-Mail: tobu@sozpsy.unizh.ch

\section{Literatur}

1. ButlerRN, LewisMI (1996) Alte Lieberostetnicht. Über den Umgang mit Sexualität im Alter. Huber, Bern

2. http://www.who.int/reproductive-health/gender/ sexual_health.html (retrieved 31.10.2005)

3. Schmidt G, Matthiesen S (2003) Spätmoderne 60Jährige. BZgA Forum 1: 16-24

4. Bucher T, Hornung R, Buddeberg C (2003) Sexualität in der zweiten Lebenshälfte. Ergebnisse einer empirischen Untersuchung. ZSexualforschung 16: 249-270

5. von Sydow K (1991) Psychosexuelle Entwicklung im Lebenslauf. Eine biographische Studie bei Frauen der Geburtsjahrgänge 1895-1935. Roderer, Regensburg

6. Bucher T, Hornung R, Gutzwiller F, Buddeberg C (2001) Sexualität in der zweiten Lebenshälfte. Erste Ergebnisse einer Studie in der deutschsprachigen Schweiz. In: Berberich H, Brähler E (Hrsg) Sexualität und Partnerschaft in der zweiten Lebenshälfte. Psychosozial-Verlag, Giessen, S 31-59

7. Hauser G (1997) Neue Bewertungsskala für das klimakterische Syndrom (Menopause Rating Scale, MSR). Schweiz Med Wochenschr 127: 122-127

8. Schultz-Zehden B (2004) Das Klimakterium als Herausforderung. Psychotherapeut 49: 350-356

9. Maake C (2005) Biologische Grundlagen der Sexualität. In: Buddeberg C (Hrsg) Sexualberatung. Eine Einführung für Ärzte, Psychotherapeuten und Familienberater. Thieme, Stuttgart, S 19-29

10. Rosemeier HP, Zerdick Y (1995) Intimität und Klimakterium. Psychomed 7: 223-226

11. Marcus-Newhall A, Thompson S (2001) Examining a gender stereotype: menopausal women. J Applied Social Psychol 31: 698-719

12. Rosemeier H (2001) Zur Psychologie des Klimakteriums. In: Berberich $\mathrm{H}$, Brähler E (Hrsg) Sexualität und Partnerschaft in der zweiten Lebenshälfte. Psychosozial-Verlag, Gießen, S 61-85

13. Cain VS, Johannes CB, Avis NE et al. (2003) Sexual functioning and practices in a multi-ethnic study of midlife women: Baseline results from SWAN. J Sex Res 40: 266-276

14. Deeks A, McCabe MP (2001) Sexual function and the menopausal women: the importance of age and partner's sexual functioning. J Sex Res 38: 219-225

15. Hartmann U, Philippson S, Heiser K, Rüffer-Hesse C (2004) Low sexual desire in midlife and older women: personality factors, psychosocial development, present sexuality. Menopause. J. North Am Menopause Society 11: 726-740

16. Schultz-Zehden B (1998) Frauengesundheit in und nach den Wechseljahren. Die 1000 Frauen-Studie. Kempkes, Gladenbach

17. Schiavi RC (1999) Aging and male sexuality. Cambridge University Press, Cambridge

18. Feldman HA, Goldstein I, Hatzichristou DG et al. (1994) Impotence and its medical and psychosocial correlates: results of the Massachusetts Male Aging Study. J Urol 151: 54-61
19. Ludwig G (2001) Endokrine Veränderungen beim älteren Mann aus urologischer Sicht. In: Berberich $H$, Brähler E (Hrsg) Sexualität und Partnerschaft in der zweiten Lebenshälfte. Psychosozial-Verlag, Gießen S 159-166

20. Schatzl G, Brössner C, Schmid S et al. (2000) Endocrine status in elderly men with lower urinary tract symptoms: correlation of age, hormonal status, and lower urinary tract function. Urology 55: 397-402

21. Bucher T (2003) Sexualität in der zweiten Lebenshälfte: Alter - Partnerschaft - Zufriedenheit. In: Hornung R, Buddeberg C, Bucher T (Hrsg) Sexualität im Wandel. vdf Hochschulverlag, Zürich, S 195-208

22. Schmidt G, Matthiesen S, Meyerhof U (2004) Alter Beziehungsform und Beziehungsdauer als Faktoren sexueller Aktivität in heterosexuellen Beziehungen. Eine empirische Studie an drei Generationen. Z Sexualforschung 17: 116-133

23. Buddeberg $C$ (2005) Sexualberatung. Eine Einführung für Ärzte, Psychotherapeuten und Familienberater. Thieme, Stuttgart

24. Fröhlich G (2001) Die Behandlung von Sexualstörungen in der urologischen Praxis unter Berücksichtigung psychosomatischer und partnerschaftlicher Aspekte. In: Berberich H, Brähler E (Hrsg) Sexualität und Partnerschaft in der zweiten Lebenshälfte. Psychosozial-Verlag, Gießen, S 139-156

25. DeLamater JD, Sill M (2005) Sexual desire in later life. J Sex Res 42: 138-149

26. Laumann EO, Nicolosi A, Glasser DB et al. (2005) Sexual problems among women and men aged 4080: prevalence and correlates identified in the Global Study of Sexual Attitudes and Behaviours. Int J Impotence Res 17: 39-57

27. Pfeiffer E, Verwoerdt A, Davis GC (1974) Sexual behaviour in Middle Life. In: Palmore E (ed) Normal aging II. Reports from the Duke Longitudinal Studies, 1970-1973. Duke University Press, Durham, pp 243-251

28. Klaiberg A, Brähler E, Schumacher J (2001) Determinanten der Zufriedenheit mit Sexualität und Partnerschaft in der zweiten Lebenshälfte. In: Berberich $\mathrm{H}$, Brähler E (Hrsg) Sexualität und Partnerschaft in der zweiten Lebenshälfte. PsychosozialVerlag, Gießen S 105-127

29. Byers SE (2005) Relationship satisfaction and sexual satisfaction: a longitudinal study of individuals in long-term relationships. J Sex Res 42: 113-118

30. Bucher T (2002) Sexualität und Partnerschaft in der zweiten Lebenshälfte. Ein kausalanalytisches Strukturgleichungsmodell zum Einfluss von Beziehungsfaktoren auf das sexuelle Interesse, die sexuelle Aktivität und Zufriedenheit bei heterosexuellen Menschen ab 45 Jahren. Dissertation an der Philosophischen Fakultät der Universität Zürich

31. Baumeister R (2000) Gender differences in erotic plasticity: the female sex drive as socially flexible and responsive. Psychological Bull 126: 347-374

32. Basson R (2000) The female sexual response: a different model. J Sex Marital Ther 26: 51-65

33. Basson R (2004) Recent advances in women's sexual function and dysfunction. Menopause. Journal North Am Menopause Society 11: 714-725

34. Katz S, Marshall B (2003) New sex for old: lifestyle, consumerism, and the ethics of aging well. J Aging Studies 17: 3-16

35. Braun M, Klotz T, Engelmann U (2004) Männliche Sexualität und Alter. Thieme, Stuttgart

36. Ponholzer A, Roehlich M, Racz U et al. (2005) Female sexual dysfunction in a healthy Austrian cohort: prevalence and risk factors. Eur Urol 47: 366-375

37. Tiefer $L$ (2001) A new view of women's sexual problems: why new? why now? J Sex Res 38: 89-96 